\title{
Hybrid Salp Swarm and Differential Evolution for Optimal Power Flow
}

\author{
Harish Kumar \\ Gurgaon Institute of Technology \& Management, \\ Bilaspur-Tauru Road, Gurugram, Haryana \\ harishkumarkhola@gmail.com
}

\begin{abstract}
Optimal Power Flow (OPF) issue is a non-linear optimization problem that was extensively exploited in power system operations. As a consequence of these characteristics, resolving the OPF issue is a well-liked and demanding task in optimizing power systems. Recently, a lot of developed optimization algorithms are used to pact with the OPF crisis. Nevertheless, the majority of the techniques are unimpeded. In this work, Hybrid Salp Swarm and differential evolution with the self-adaptive penalty (HSSDE-SP) is introduced to attain the optimum solution for the power flow issue. To confirm the efficiency of the developed technique, simulations were carried out on the IEEE 30-bus test system that integrates solar energy and wind energy with thermal generators. The experimentation outcomes show the superiority of the developed technique.
\end{abstract}

Keywords: OPF; Power System; IEEE Bus System; Solar Energy; Wind Energy; Optimization Algorithms

\begin{tabular}{ll} 
Nomenclature & \\
\hline Abbreviations & Descriptions \\
\hline REGs & Renewable Energy Generations \\
RES & Renewable Energy Source \\
HBMO & Honey Bee Mating Optimization \\
BSSs & Battery Storage Systems \\
MHBMO & Modified Honey Bee Mating Optimization \\
ED & Economic Dispatch \\
TS & Tabu Search \\
JADE & Enhanced Adaptive Differential Evolution \\
MPA & Mathematical Programming Algorithm \\
SCOPF & Security Constrained Optimal Power Flow \\
GSA & Gravitational Search Algorithm \\
ICA & Imperialist Competitive Algorithm \\
PSO & Particle Swarm Optimization \\
ABCA & Artificial Bee Colony Algorithm \\
ISSO & Improved Social Spider Optimization Algorithm \\
MICA & Modified Imperialist Competitive Algorithm \\
QOMJaya & Quasi-Oppositional Modified Jaya \\
MSA & Moth Swarm Algorithm \\
HTW & Hydrothermal- Wind \\
ESDE-MC & Enhanced Self-adaptive DE with Mixed Crossover \\
MSA & Moth Swarm Algorithm \\
SKHA & Stud Krill Herd Algorithm \\
DSA & Differential Search Algorithm \\
SFLA & Shuffle Frog Leaping Algorithm \\
ICBOA & Improved Colliding Bodies Optimization Algorithm \\
IABC & Improved Artificial Bee Colony \\
DE & Differential Evolution \\
CHP & Combined heat and power \\
MOOPF & multi-objective OPF \\
IELMA & Improved Electromagnetism-Like Mechanism Algorithm \\
& \\
\hline &
\end{tabular}

(C) Resbee Publishers 


\begin{tabular}{ll}
\hline SP & SELF-ADAPTIVE PENALTY \\
COA & Cuckoo Optimization Algorithm \\
DNs & Distribution Networks \\
KHA & Krill Herd Algorithm \\
GBBICA & Gaussian Bare-Bones Imperialist Competitive Algorithm \\
CH & Constraint Handling \\
APFPA & Adaptive Flower Pollination Algorithm \\
GWO & Grey Wolf Optimizer \\
MSFLA & Modified Shuffle Frog Leaping Algorithm \\
PF & Pareto front \\
\hline
\end{tabular}

\section{Introduction}

In the power systems operation, OPF plays an extensive role [1]. Generally, OPF schedules the power system decision variables in the best manner that concurrently assures power flow balance equations and power system constraints (for instance., apparent power and nodal voltages in the feeders). The ensuing optimization issue is typically large-scale non-convex and with mixed-integer variables. Several techniques have been suggested resolving the problem of OPF for diverse kinds of voltage levels, network topologies, without or with REGs and embedded BSSs. Over the past decades, these techniques were evaluated comprehensively in several review papers on OPF [24].

At first, the OPF was envisaged as an addition of the traditional ED, whereas the ED and power flow issues are resolved concurrently. This untimely technique of OPF can be devised as a nonlinear issue whose objective model is to decide the control variables, and state variables which reduce the generation costs, cause to experience the power balance equation and the transmission network constraints to make sure the double objectives of economic and safe system operation [23].

As a result, OPF has received more consideration because of the raise of RES in the network. The crisis of $\mathrm{OPF}$ is typified as nonlinear, multidimensional, and a non-convex optimization problem. OPF comprises deciding a stable operating point which reduces the emission of gases and generated electric power cost whilst fulfilling operating limits and meeting demand. Resolving OPF problems have turned out to be greatly complicated with widespread inclusion of stochastic RES, like solar energy and wind power. The amplified size and network complexity, and the additional reservations to the power production, predict, bring novel confronts for each day operation and management the power grid

A large number of meta-heuristic approaches which were explained above are innovative techniques and enhanced techniques. Besides the application of these techniques, other techniques have also been used for resolving OPF issue like HBMO, MHBMO, TS, MPA, GSA, MICA, ABCA, COA, GBBICA, GWO, MSFLA, IELMA, ICBOA, SFLA, DSA, SKHA, and MSA. Amid these techniques, TS is the oldest technique that was exploited to numerous optimization issues in electrical engineering; on the other hand, the technique has not exhibited possible search capability as the application of the technique was uncomplicated for one system with 30 buses and few cases [25] [26].

Several optimization approaches were used to resolve the OPF using emission issues with or without RES. The traditional techniques namely quadratic programming, Gradient's technique, and so on was used in literature to resolve the OPF issue. These techniques undergo a few downsides like local minima trapping, dimensional curses, and a number of the hypothetical supposition that does not assurance to obtain the large-scale optimal solutions [10] [27].

Here, the most important purpose is to present the HSSDE for optimum power flow. In the proposed method, 4 enhancements are exploited for enhancing the performance of the adopted scheme. Moreover, the performance of the proposed method is shown by deploying it to optimize several objectives of the OPF crisis. Finally, it is found that the developed technique can be an effectual choice for the OPF problem.

\section{Literature Review}

In 2019, Shuijia Li et al [1], developed the EJADE-SP technique that attained the best solution for the OPF issue. In 2020, Hossein Saberi et al [2], worked on the SCOPF issue with DC load flow equations was comprehensive to regard as the transient stability margin of the generating units as a heuristic decomposition method. In 2019, Jalel Ben Hmida et al [3], proposed an ICA to resolve the OPF predicaments. In 2019, Thang Trung Nguyen [4], developed an ISSO to solve the OPF issues by optimizing power loss, fuel price, voltage deviation and contaminated emissions. In 2019, Ernest Benedito et al [5], worked on the port-Hamiltonian formalism for the OPF problem of a DC network. In 2019, Ehsan Naderi et al [6] introduced a PSO technique for the OPF problem which was combined with 
practical constraints indicated above and FACTS devices. In 2019, Ehab and Salah [7] presented a JA approach to resolve the OPF problem integrating RES using four diverse objective functions. In 2017, Xiaohui Yuan et al [8], worked on an enhanced strength Pareto evolutionary method, which was used to resolve the multi-objective OPF problem. In 2018, Erfan Mohagheghi et al [9], developed a novel reconciliation method to make sure both the possibility and optimality of comprehends operation approach. The applicability of the developed model was exhibited by exploiting a medium voltage DNs. In 2018, Partha P. Biswas et al [10], presented proper CH methods and SP and a collection of these $2 \mathrm{CH}$ methods with DE being the essential search method, on the OPF problem. In 2018, Yinliang Xu et al [11], developed a completely distributed solution to DC OPF with congestion management. The aim was to make the most of the social welfare, whilst controlling the supply-demand balance and alleviating transmission line congestion.

In 2017, Harish Pulluri et al [12], developed an ESDE-MC method to resolve the multiobjective OPF problems using contradictory objectives that imitate the minimization of emission pollution, total production cost, Lindex, and active power loss. In 2018, Warid Warid et al [13], introduced a QOMJaya to resolve diverse MOOPF problems. In 2016, Jadhav and Bamane [14], worked using the ABC approach to resolve the OPF and temperature-dependent OPF. In 2018, Attia et al [15], developed the application of a new method that was based on the enhanced Sine-Cosine algorithm to solve the OPF issue. It was an extremely coupled non-linear constrained optimization issue. In 2017, Ambarish et al [16], worked on the integrated operation of the HTW system that was devised in the OPF model. The aim was to find out the best generation scheduler with minimized loss through stressed and normal system operations. In 2017, Wenlei et al [17], worked on an enhanced heuristic method, the IABC to OPF issue in electric power grids. In 2016, Belkacem Mahdad Srairi [18], worked on a flexible planning approach for power system by exploiting a new population-based meta-heuristic approach called APFPA. The developed power system planning approach implemented and effectively applied to solve the security OPF taking into consideration faults at the significant generating unit. In 2017, Adhvaryyu et al [19] developed a maiden formulation for resolving the issues that occurred due to OPF in the power system by linking CHP. Moreover, KHA approach was used to minimize the production cost, whilst sustaining voltage at every bus and fulfilling the entire constraints. In 2017, Mohamed et al [20] developed a new MSA, to resolve the OPF issue. In addition, Lévy-mutation was developed to enhance exploitation and exploration capability, correspondingly.

\section{Formulation of OPF}

The OPF issue intends to attain the optimum settings of control parameters to minimize the chosen objective model. Arithematically, the OPF issue can be devised and it is represented as below:

$$
\begin{aligned}
& \operatorname{Min} J(a, b) \\
& s: t \quad K(a, b)=0
\end{aligned}
$$

$$
\mathrm{L}(\mathrm{a}, \mathrm{b}) \leq 0
$$

In eq. (1), $\mathrm{J}(\mathrm{a}, \mathrm{b})$ indicates the objective model; $\mathrm{K}(\mathrm{a}, \mathrm{b})$ indicates the equality constraints, and $\mathrm{L}(\mathrm{a}, \mathrm{b})$ indicates inequality constraints; $a, b$ indicate the control and state variables, correspondingly.

\subsection{State and Control Variables}

The control parametersa, a vector which comprises of real power outputs and it controls the OPF equations in eq. (2).

$$
\mathrm{a}=\left[\mathrm{A}_{\mathrm{G}_{2}}, \ldots ., \mathrm{A}_{\mathrm{G}_{\mathrm{NG}}}, \mathrm{M}_{\mathrm{G}_{2}}, \ldots ., \mathrm{M}_{\mathrm{G}_{\mathrm{NG}}}, \mathrm{S}_{\mathrm{G}_{2}}, \ldots ., \mathrm{S}_{\mathrm{G}_{\mathrm{NC}}}, \mathrm{B}_{1}, \ldots \ldots . \mathrm{B}_{\mathrm{NT}}\right]
$$

In eq. (2), " $\mathrm{A}_{\mathrm{G}_{\mathrm{i}}}$ indicates $\mathrm{i}^{\text {th }}$ bus generator active power apart from the slack bus $\mathrm{A}_{\mathrm{G}_{1}} ; \mathrm{M}_{\mathrm{G}_{\mathrm{i}}}$ indicates the voltage magnitude at $\mathrm{i}^{\text {th }}$ generator bus ( $\mathrm{P}-\mathrm{V}$ buses); $\mathrm{B}_{\mathrm{k}}$ represents the $\mathrm{k}^{\text {th }}$ branch transformer tap; $\mathrm{S}_{\mathrm{G}_{\mathrm{j}}}$ indicates the shunt compensation at $\mathrm{j}^{\text {th }}$ bus; and $\mathrm{NC}$ indicates the number of shunt compensators units, NG indicates the number of generators buses, NT indicates the number of regulating transformers", correspondingly.

Also, the state variables that explain the condition of an electrical system are shown by $b$ and it is exhibited in eq. (3).

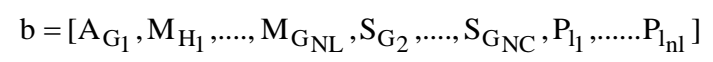


In eq. (3), $\mathrm{S}_{\mathrm{G}_{\mathrm{i}}}$ signifies the reactive power outputs at $\mathrm{i}^{\text {th }}$ generator bus; $\mathrm{M}_{\mathrm{H}_{\mathrm{m}}}$ signifies the voltage magnitude at $\mathrm{m}^{\text {th }}$ load bus; $\mathrm{P}_{\mathrm{l}_{\mathrm{n}}}$ signifies the $\mathrm{n}^{\text {th }}$ line loading; $\mathrm{NL}$ signifies the load bus number, and $n l$ signifies transmission lines, correspondingly.

\subsection{Constraints}

The equality and inequality parameters should be fulfilled for resolving the OPF problem.

The balanced amid reactive and active power is representative of the equality constraints that are devised as below:

$$
\begin{aligned}
& A_{G_{i}}-A_{D_{i}},-M_{i} \sum_{j=1}^{N B} M_{j}\left[K_{i j} \cos \left(\delta_{i}-\delta_{j}\right)+X_{i j} \sin \left(\delta_{i}-\delta_{j}\right)\right]=0, i=1, \ldots, N B \\
& S_{G_{i}}-S_{D_{i}},-M_{i} \sum_{j=1}^{N B} M_{j}\left[K_{i j} \cos \left(\delta_{i}-\delta_{j}\right)-X_{i j} \sin \left(\delta_{i}-\delta_{j}\right)\right]=0, i=1, \ldots, N B
\end{aligned}
$$

In eq. (4) $\delta_{\mathrm{i}}$ indicates the $\mathrm{i}^{\text {th }}$ bus voltage angle; $\mathrm{A}_{\mathrm{D}_{\mathrm{i}}}$ indicates the active load demands; $\mathrm{NB}$ indicates the number of buses; $\mathrm{M}_{\mathrm{D}_{\mathrm{i}}}$ indicates the reactive load demands; and $\mathrm{X}_{\mathrm{ij}}, \mathrm{K}_{\mathrm{ij}}$ indicates transfer susceptance and conductance among bus $\mathrm{i}$ and $\mathrm{j}$, correspondingly.

\section{(i) Generator constraints:}

$$
\begin{aligned}
& \mathrm{A}_{\mathrm{G}_{\mathrm{i}}}^{\min } \leq \mathrm{A}_{\mathrm{G}_{\mathrm{i}}} \leq \mathrm{A}_{\mathrm{G}_{\mathrm{i}}}^{\max }, \mathrm{i}=1, \ldots \ldots ., \mathrm{NG} \\
& \mathrm{S}_{\mathrm{G}_{\mathrm{i}}}^{\min } \leq \mathrm{S}_{\mathrm{G}_{\mathrm{i}}} \leq \mathrm{S}_{\mathrm{G}_{\mathrm{i}}}^{\max }, \mathrm{i}=1, \ldots \ldots ., \mathrm{NG} \\
& \mathrm{M}_{\mathrm{G}_{\mathrm{i}}}^{\min } \leq \mathrm{M}_{\mathrm{G}_{\mathrm{i}}} \leq \mathrm{M}_{\mathrm{G}_{\mathrm{i}}}^{\max }, \mathrm{i}=1, \ldots \ldots ., \mathrm{NG}
\end{aligned}
$$

In eq. (6), $\mathrm{A}_{\mathrm{G}_{\mathrm{i}}}, \mathrm{S}_{\mathrm{G}_{\mathrm{i}}}$, and $\mathrm{M}_{\mathrm{G}_{\mathrm{i}}}$ ought to lie among its relevant upper limits $\left(\mathrm{A}_{\mathrm{G}_{\mathrm{i}}}^{\max }, \mathrm{S}_{\mathrm{G}_{\mathrm{i}}}^{\max }, \mathrm{M}_{\mathrm{G}_{\mathrm{i}}}^{\max }\right)$ and lower $\left(\mathrm{A}_{\mathrm{G}_{\mathrm{i}}}^{\mathrm{min}}, \mathrm{S}_{\mathrm{G}_{\mathrm{i}}}^{\mathrm{min}}, \mathrm{M}_{\mathrm{G}_{\mathrm{i}}}^{\mathrm{min}}\right)$.

\section{(ii) Shunt compensator constraints:}

(iii) Transformer constraints:

$$
\mathrm{S}_{\mathrm{C}_{\mathrm{j}}}^{\min } \leq \mathrm{S}_{\mathrm{C}_{\mathrm{j}}} \leq \mathrm{S}_{\mathrm{C}_{\mathrm{j}}}^{\max }, \mathrm{j}=1, \ldots \ldots, \mathrm{NC}
$$

$$
\mathrm{B}_{\mathrm{k}}^{\min } \leq \mathrm{B}_{\mathrm{k}} \leq \mathrm{B}_{\mathrm{k}}^{\max }, \mathrm{k}=1, \ldots \ldots, \mathrm{NT}
$$

(iv)Security constraints:

$$
\begin{aligned}
& \mathrm{S}_{\mathrm{H}_{\mathrm{m}}}^{\min } \leq \mathrm{S}_{\mathrm{H}_{\mathrm{m}}} \leq \mathrm{S}_{\mathrm{H}_{\mathrm{m}}}^{\max }, \mathrm{m}=1, \ldots \ldots ., \mathrm{NL} \\
& \mathrm{P}_{\mathrm{l}_{\mathrm{n}}} \leq \mathrm{P}_{\mathrm{l}_{\mathrm{n}}}^{\max }, \mathrm{n}=1, \ldots \ldots ., \mathrm{nl}
\end{aligned}
$$

\subsection{Objective function}

As aforesaid, an objective model requires choosing the optimal objective. Here, five diverse objectives were chosen for optimization and diverse objectives include diverse purposes as exhibited in fig .1. For instance, the generation cost minimization is to store the power system cost; the real power loss minimization is to minimize the losses in transmission; minimize the voltage deviation and to enhance voltage quality; the emission minimization is to minimize the environmental pollution; and emission and generation cost minimization is to store the power system cost and it considers environmental pollution. The comprehensive objective models are detailed are as follows: 


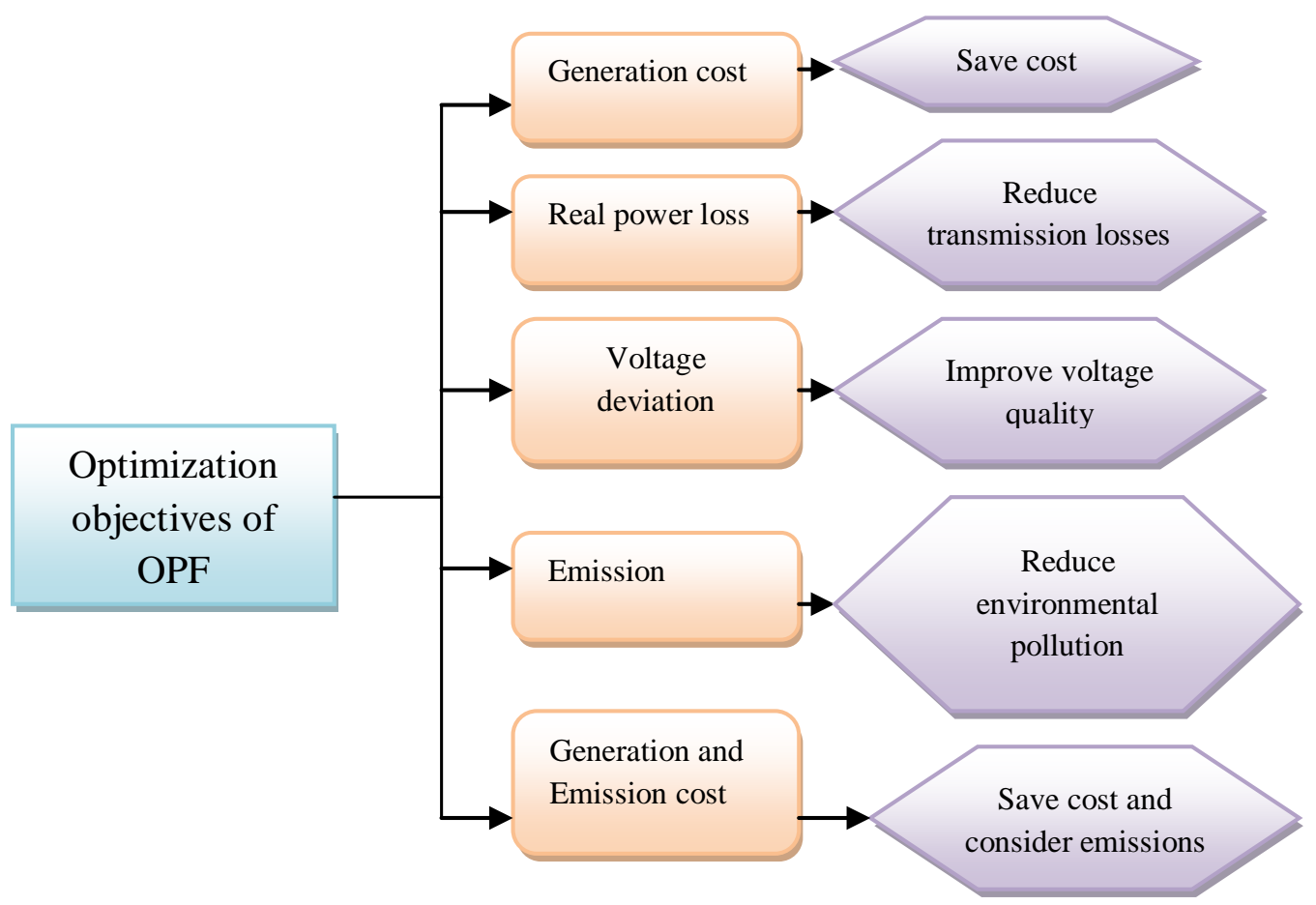

Fig. 1. Schematic diagram of optimization objectives of $O P F$

Case 1: Minimization of Generation cost

The total generation cost considering the value loading effect is defined as follows:

$$
\mathrm{J}_{\mathrm{C}}=\sum_{\mathrm{i}=1}^{\mathrm{NG}} \mathrm{x}_{\mathrm{i}}+\mathrm{y}_{\mathrm{i}} \mathrm{A}_{\mathrm{G}_{\mathrm{i}}}+\mathrm{z}_{\mathrm{i}} \mathrm{A}^{2} \mathrm{G}_{\mathrm{i}}+\left|\mathrm{d}_{\mathrm{i}} \cdot \sin \left(\mathrm{e}_{\mathrm{i}} \cdot\left(\mathrm{A}_{\mathrm{G}_{\mathrm{i}}}^{\mathrm{min}}-\mathrm{A}_{\mathrm{G}_{\mathrm{i}}}\right)\right)\right|
$$

In eq. (13), $e_{i}$ and $\left(x_{i}, y_{i}, z_{i}\right)$ indicates the cost coefficient of $i^{\text {th }}$ generator by means of loading effect and $\mathrm{d}_{\mathrm{i}}$ in that order.

Case 2: Minimization of Real power loss

The objective function of real power loss is stated in eq. (14).

$$
\mathrm{J}_{\text {loss }}=\sum_{\mathrm{i}=1}^{\mathrm{nl}} \sum_{\mathrm{j} \neq \mathrm{i}}^{\mathrm{nl}} \mathrm{K}_{\mathrm{ij}}\left[\mathrm{M}_{\mathrm{i}}^{2}+\mathrm{M}_{\mathrm{j}}^{2}-2 \mathrm{M}_{\mathrm{i}} \mathrm{M}_{\mathrm{j}} \cos \left(\delta_{\mathrm{i}}-\delta_{\mathrm{j}}\right)\right]
$$

Case 3: Voltage deviation minimization

In the power network, voltage deviation defines the voltage quality, and it is represented in eq. (15).

$$
\mathrm{J}_{\mathrm{VD}}=\sum_{\mathrm{m}=1}^{\mathrm{NL}}\left|\mathrm{V}_{\mathrm{Lm}}-1.0\right|
$$

Case 4: Emission minimization

In recent times, the emission has achieved more concentration and it is evaluated as in Eq. (16).

$$
\mathrm{J}_{\mathrm{E}}=\sum_{\mathrm{I}=1}^{\mathrm{NG}} \alpha_{\mathrm{i}}+\beta_{\mathrm{i}} \mathrm{A}_{\mathrm{G}_{\mathrm{i}}}+\gamma_{\mathrm{i}} \mathrm{A}^{2} \mathrm{G}_{\mathrm{i}}+\omega_{\mathrm{i}} \mathrm{e}^{\left(\mathrm{v}_{\mathrm{i}} \mathrm{A}_{\mathrm{G}_{\mathrm{i}}}\right)}
$$

In eq. (16), $\alpha_{\mathrm{i}}, \beta_{\mathrm{i}}, \gamma_{\mathrm{i}}, v_{\mathrm{i}}, \omega_{\mathrm{i}}$ indicates the coefficients of emission equivalent to $\mathrm{i}^{\text {th }}$ generator.

Case 5: Emission and Generation cost minimization

The generation and emission costs are considered in this case. The objective function of the cost is represented as follows:

$$
\mathrm{J}_{\mathrm{CE}}=\mathrm{J}_{\mathrm{C}}+\mathrm{C}_{\mathrm{t}} \cdot \mathrm{J}_{\mathrm{E}}
$$

In eq. (17), $C_{t}$ indicates the carbon tax, and its price, is fixed as $20(\$ / h)$.

\section{Optimized Proposed Hybrid Salp Swarm-Differential}

Fig. 2 depict the flowchart of the developed model that depends on the integration among the Salp Swarm Algorithm [22] and DE [21]. The steps involved in the developed algorithm are discussed below for a varied number of iteration $\mathrm{t}_{\max }$. 
The population is generated arbitrarily, and the objective functions are calculated. The developed algorithm initially runs based on the hybridized method for all iteration. In the proposed algorithm, DE improves the feature exploitation ability of SS. The developed technique verified if the termination condition has been met. Here, 3 important phases are carried out on every salps location: initialization, salp position updating by adopted method, and updating the archive to decide on the approximation to $\mathrm{PF}$.

\subsection{Initialization Phase}

In this phase, the population $\mathrm{Y}$ is initialized.

The inputs are $\mathrm{N}, \mathrm{d}, \mathrm{lb}$, and $\mathrm{ub}$, which point out the size of the population, the dimension of issues, lower and upper bounds respectively. Consequently, the population is assigned as shown in Eq. (18), where $\mathrm{rn}(\mathrm{N}, \mathrm{d})$ point out the uniformly distributed arbitrary integer.

$$
\mathrm{Y}=\mathrm{rn}(\mathrm{N}, \mathrm{d}) \times(\mathrm{ub}-\mathrm{lb})+\mathrm{lb}
$$

Moreover, the non-dominated solutions are determined and archive $A_{R}$ gets updated.

\subsection{Population Update Exploiting Developed Method}

Generally, HSSADE initiates by choosing the optimal solution and by calculating the fitness value for every solution. The procedure of choosing the optimal solution depends on the optimal objective function. Accordingly, a single non-dominated solution is chosen as the optimum solution $\mathrm{y}_{\mathrm{b}}$. The roulette-wheel method is exploited for selecting $\mathrm{y}_{\mathrm{b}}$ as in eq. (19).

$$
\mathrm{P}_{\mathrm{sel}}=\mathrm{C} \times \mathrm{N}_{\mathrm{seg}}
$$

In eq. (19), $\mathrm{N}_{\mathrm{seg}}$ and $\mathrm{C}>1$ indicate the number of Pareto optimal solutions of the $\mathrm{i}^{\text {th }}$ segment, and a constant, correspondingly.

Subsequently, the probability Probis calculated for all solutions regarding the value of 1 st objective function using eq. (20):

$$
P_{i}=\frac{f_{1}}{\sum_{i=1}^{N} f_{1}}
$$

Subsequently, the present solution $y_{i}$ is updated using SSA or DE based on $y_{b}$ and $P_{i}$. For instance, if $P_{i}>c$ (whereas $P_{i} \in[0,1]$; the important suitable values of the threshold $\beta$ were attained be 0.65), subsequently the SSA operator is exploited for updating $\mathrm{y}_{\mathrm{i}}$; else, DE operators are exploited for updating $y_{i}$. Hence, in the scenario which $P_{i}$ is lesser than $\beta$, it represents that the present solution $y_{i}$ gets attracted to a stagnation point. The objective function for every solution is updated using Eq.(13), (14), (15), (16), and (17).

whereas $\mathrm{F}_{1}=\left[\mathrm{f}_{1}, \mathrm{f}_{2}\right]^{\mathrm{T}}$ and have to fulfill $\left.-8 \leq \mathrm{S} \leq 8\right)$.

\subsection{Update the archive}

To find out non-dominated solutions, the density estimation information is exploited for controlling population diversities. To decide on those solutions, the technique measures the neighboring solution's number using a particular distance, which is computed as per eq. (21).

$$
\mathrm{D}=\frac{\max \left(\mathrm{f}_{\mathrm{i}}\right)-\min \left(\mathrm{f}_{\mathrm{i}}\right)}{|\mathrm{AR}|} \mathrm{i}=1,2,
$$

In eq. (21), $|\mathrm{AR}|$ point out the archive size. The solutions $\mathrm{Y}$ were updated based on updated AR by electing the optimal $\mathrm{N}$ solutions from it. Consequently, the remaining solutions are select from the next front.

\subsection{A complete description of the HybridSSADE approach}

The important phases of the developed algorithm have been developed: initialization, solution update based on hybrid SSADE approach, and AR update. 


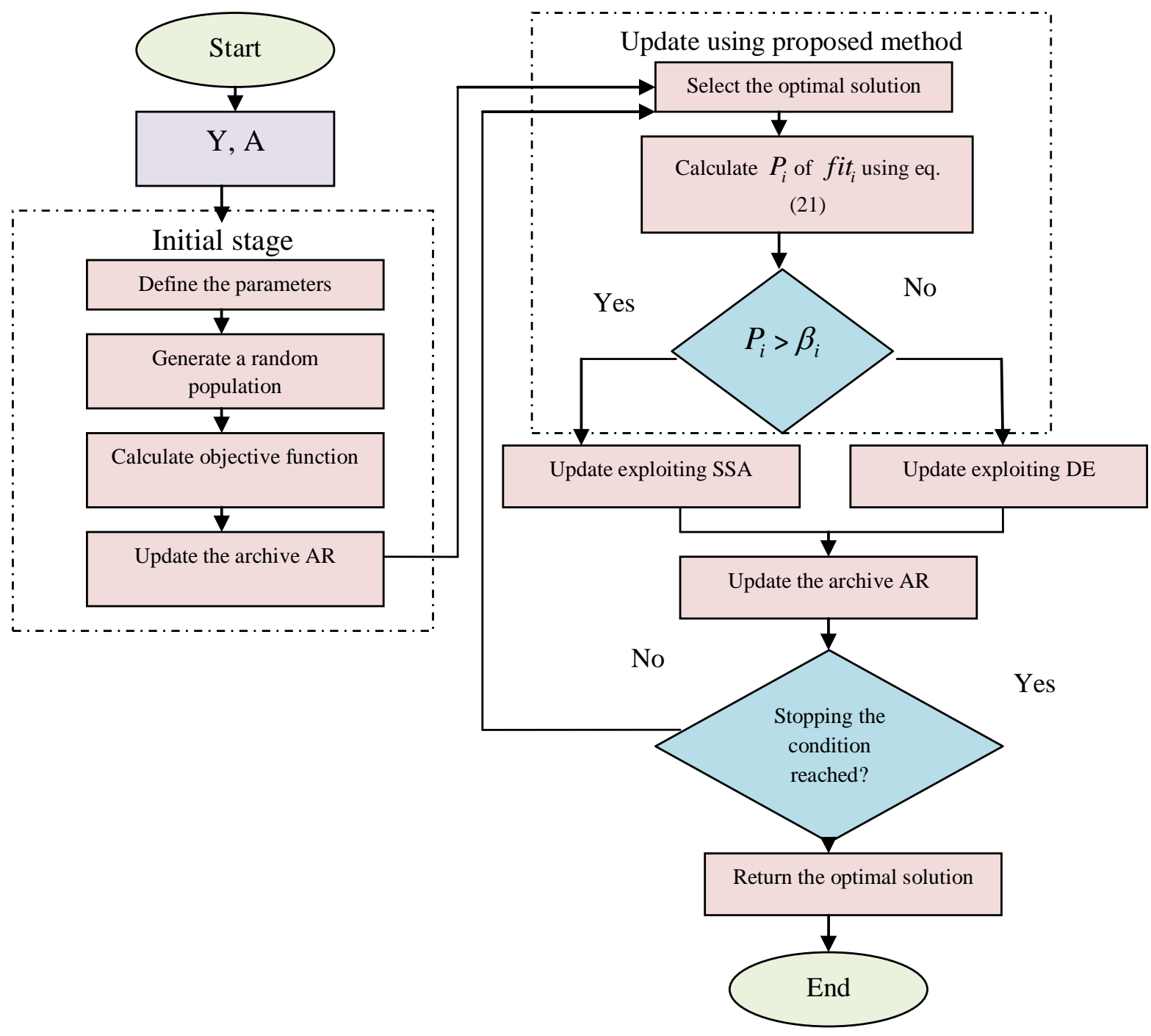

Fig. 2. Flow chart of the adopted model

\section{Results and Discussions}

\subsection{Experimental Procedure}

The performance of the developed model was examined on the IEEE 30-bus system. Here, the conventional thermal generators on the 5th bus and 11th bus were restored using wind generators, and bus 13 is restored by solar generators.

Moreover, the adopted approach was compared over conventional schemes such as PSO, SFDE, JADE, SP-DE, TLBO, and SHADE-SF.

\subsection{Statistical Comparision}

A statistical evaluation of the experimentation outcomes for the selected test system is described for five cases exhibiting "maximum (Max), minimum (Min), average (Mean) cost, standard deviation (Std), and pvalue" as produced by all evaluated methods.

Table 1, 2, 3, 4, and 5 shows the statistical analysis of the adopted and existing algorithms on case 1, 2, 3, 4, and 5. From the evaluated methods, it was observed that the conventional algorithms have robustness when the proposed method demonstrates better performance on the accuracy while comparing with other methods. 
Table 1: Statistical analysis of proposed and conventional approaches on case 1

\begin{tabular}{llllll}
\hline Methods & Min & P value & Max & Std & Mean \\
SF-DE & 822.227 & $2.93 \mathrm{e}^{-02}$ & 822.2567 & $8.62 \mathrm{e}^{-03}$ & 822.2362 \\
TLBO & 822.6233 & $2.86 \mathrm{e}^{-09}$ & 822.9008 & $2.07 \mathrm{e}^{-01}$ & 822.6236 \\
ECHT-DE & 822.227 & $3.76 \mathrm{e}^{-03}$ & 822.2666 & $7.62 \mathrm{e}^{-03}$ & 822.2392 \\
SP-DE & 822.227 & $9.30 \mathrm{e}^{-03}$ & 822.2662 & $8.22 \mathrm{e}^{-03}$ & 822.2372 \\
JADE & 822.227 & - & 822.2639 & $6.62 \mathrm{e}^{-03}$ & 822.232 \\
SHADE-SF & 822.227 & - & 822.2639 & $5.22 \mathrm{e}^{-03}$ & 822.229 \\
Proposed method & 822.2226 & - & 822.2639 & $8.66 \mathrm{e}^{03}$ & 822.2362 \\
\hline
\end{tabular}

Table 2: Statistical analysis of proposed and conventional approaches on case 2

\begin{tabular}{llllll} 
Methods & Min & Max & P value & Std & Mean \\
\hline SF-DE & 3.3335 & 3.8668 & $1.86 \mathrm{e}^{-09}$ & $1.60 \mathrm{e}^{-01}$ & 3.6377 \\
TLBO & 3.136 & 3.3075 & $1.86 \mathrm{e}^{-09}$ & $3.98 \mathrm{e}^{-01}$ & 3.1158 \\
ECHT-DE & 3.0739 & 3.1669 & $1.63 \mathrm{e}^{-01}$ & $1.73 \mathrm{e}^{-02}$ & 3.0861 \\
SP-DE & 3.0733 & 3.1086 & - & $1.35 \mathrm{e}^{-02}$ & 3.0836 \\
JADE & 3.0733 & 3.106 & $1.35 \mathrm{e}^{-01}$ & $1.60 \mathrm{e}^{-02}$ & 3.0866 \\
SHADE-SF & 3.0733 & 3.1039 & - & $1.03 \mathrm{e}^{-02}$ & 3.0783 \\
Proposed method & 3.0733 & 3.1098 & - & $1.08 \mathrm{e}^{-02}$ & 3.0789 \\
\hline
\end{tabular}

Table 3: Statistical analysis of proposed and conventional approaches on case 3

\begin{tabular}{llllll}
\hline Methods & Min & Max & P value & Std & Mean \\
SF-DE & 0.4844 & 0.4044 & $1.84 \mathrm{e}^{-09}$ & $4.41 \mathrm{e}^{-03}$ & 0.4944 \\
TLBO & 0.444 & 0.4491 & $1.84 \mathrm{e}^{-09}$ & $5.84 \mathrm{e}^{-04}$ & 0.4445 \\
ECHT-DE & 0.4452 & 0.4458 & $5.11 \mathrm{e}^{-06}$ & $2.29 \mathrm{e}^{-04}$ & 0.4454 \\
SP-DE & 0.4452 & 0.4458 & $2.11 \mathrm{e}^{-05}$ & $2.29 \mathrm{e}^{-04}$ & 0.4454 \\
JADE & 0.4452 & 0.4458 & $2.00 \mathrm{e}^{-06}$ & $1.42 \mathrm{e}^{-04}$ & 0.4454 \\
SHADE-SF & 0.4452 & 0.4458 & $1.90 \mathrm{e}^{-03}$ & $2.48 \mathrm{e}^{-04}$ & 0.4454 \\
Proposed method & 0.4452 & 0.4458 & $1.11 \mathrm{e}^{-01}$ & $1.81 \mathrm{E} \mathrm{e}^{-04}$ & 0.4454 \\
\hline
\end{tabular}

Table 4: Statistical analysis of proposed and conventional approaches on case 4

\begin{tabular}{llllll} 
Methods & Min & P value & Max & Std & Mean \\
\hline SF-DE & 0.0868 & $1.86 \mathrm{e}^{-09}$ & 0.1047 & $2.17 \mathrm{e}^{-03}$ & 0.088 \\
TLBO & 0.0868 & $7.36 \mathrm{e}^{-04}$ & 0.0868 & $1.26 \mathrm{e}^{-05}$ & 0.0868 \\
ECHT-DE & 0.0868 & $1.48 \mathrm{e}^{-01}$ & 0.0868 & $6.73 \mathrm{e}^{-08}$ & 0.0868 \\
SP-DE & 0.0868 & $4.83 \mathrm{E}-02$ & 0.086 & $2.86 \mathrm{e}^{-05}$ & 0.0868 \\
JADE & 0.0868 & $1.48 \mathrm{e}^{-01}$ & 0.0868 & $2.01 \mathrm{e}^{-07}$ & 0.0868 \\
SHADE-SF & 0.0867 & - & 0.0868 & $2.06 \mathrm{e}^{-05}$ & 0.0868 \\
Proposed method & 0.0844 & - & 0.0868 & $3.86 \mathrm{e}^{-04}$ & 0.0867 \\
\hline
\end{tabular}

Table 5: Statistical analysis of proposed and conventional approaches on case 5

\begin{tabular}{llllll}
\hline Methods & Min & Max & P value & Std & Mean \\
SF-DE & 922.4233 & 922.9009 & $2.96 \mathrm{e}^{-09}$ & $2.07 \mathrm{e}^{-01}$ & 922.6236 \\
TLBO & 922.227 & 922.2547 & $2.93 \mathrm{e}^{-02}$ & $9.62 \mathrm{e}^{-03}$ & 922.2362 \\
ECHT-DE & 922.227 & 922.2442 & $9.30 \mathrm{e}^{-03}$ & $9.22 \mathrm{e}^{-03}$ & 922.2372 \\
SP-DE & 922.227 & 922.2446 & $3.74 \mathrm{e}^{-03}$ & $7.42 \mathrm{e}^{-03}$ & 922.2392 \\
JADE & 922.227 & 922.2439 & - & $5.22 \mathrm{e}^{-03}$ & 922.229 \\
SHADE-SF & 922.227 & 922.2439 & - & $6.42 \mathrm{e}^{-03}$ & 922.232 \\
Proposed method & 922.2226 & 922.2439 & - & $9.66 \mathrm{e}^{-03}$ & 922.2342 \\
\hline
\end{tabular}

\section{Conclusion}

A hybrid salp swarm and differential evolution optimization model, called HSSDE-SP, was developed in this work. The proposed method, four enhancements were developed to improve the performance of conventional algorithms while resolving the OPF problem. To verify the efficiency of the proposed method, it was exploited to resolve 5 diverse OPF objective models in an enhanced IEEE 30-bus test system. The outcomes attained by the proposed method were evaluated with various optimization methods. The performance analysis exhibits that the developed method was extremely competitive than conventional algorithms. Here, the proposed method was significant in minimizing the generation and emission cost. Consequently, it was an effectual choice to solve the OPF problem. 


\section{References}

[1] Shuijia Li, Wenyin Gong, Ling Wang, Xuesong Yan, Chengyu Hu, "Optimal power flow by means of improved adaptive differential evolution", Energy, Volume 1981, May 2020.

[2] Hossein Saberi, Turaj Amraee, Cuo Zhang, Zhao Yang Dong, "A heuristic benders-decomposition-based algorithm for transient stability constrained optimal power flow", Electric Power Systems Research,Volume 185,August 2020.

[3] Jalel Ben Hmida, Terrence Chambers, Jim Lee,"Solving constrained optimal power flow with renewables using hybrid modified imperialist competitive algorithm and sequential quadratic programming" Electric Power Systems Research,Volume 177,December 2019

[4] Thang Trung Nguyen,"A high performance social spider optimization algorithm for optimal power flow solution with single objective optimization",Energy,Volume 17115, March 2019,Pages 218-240.

[5] Ernest Benedito, Dunstano del Puerto-Flores, Arnau Dòria-Cerezo, Jacquelien M. A. Scherpen,"PortHamiltonian based Optimal Power Flow algorithm for multi-terminal DC networks",Control Engineering Practice,Volume 83,February 2019,Pages 141-150.

[6] Ehab E. Elattar, Salah K,"An efficient particle swarm optimization algorithm to solve optimal power flow problem integrated with FACTS devices",Applied Soft Computing,Volume 80,July 2019,Pages 243-262.

[7] ElSayed Ehsan Naderi, Mahdi Pourakbari-Kasmaei, Hamdi AbdiModified JAYA algorithm for optimal power flow incorporating renewable energy sources considering the cost, emission, power loss and voltage profile improvement,Energy,Volume 1781, July 2019Pages 598-609.

[8] Xiaohui Yuan, Binqiao Zhang, Pengtao Wang, Ji Liang, Xiaohui Lei,"Multi-objective optimal power flow based on improved strength Pareto evolutionary algorithm",Energy,Volume 1221, March 2017,Pages 70-82.

[9] Erfan Mohagheghi, Aouss Gabash, Mansour Alramlawi, Pu Li,"Real-time optimal power flow with reactive power dispatch of wind stations using a reconciliation algorithm",Renewable Energy,Volume 126,October 2018,Pages 509-523.

[10] Partha P. Biswas, P. N. Suganthan, R. Mallipeddi, Gehan A. J. Amaratunga,"Optimal power flow solutions using differential evolution algorithm integrated with effective constraint handling techniques"Engineering Applic ations of Artificial IntelligenceVolume 68February 2018Pages 81-100

[11] Yinliang Xu, Hongbin Sun, Houde Liu, Qing Fu,"Distributed solution to DC optimal power flow with congestion management", International Journal of Electrical Power \& Energy Systems,Volume 95February 2018,Pages 7382.

[12] Harish Pulluri, R. Naresh, Veena Sharma,"An enhanced self-adaptive differential evolution based solution methodology for multiobjective optimal power flow",Applied Soft Computing,Volume 54,May 2017,Pages 229245.

[13] Warid Warid, Hashim Hizam, Norman Mariun, Noor Izzri Abdul Wahab,"A novel quasi-oppositional modified Jaya algorithm for multi-objective optimal power flow solution",Applied Soft Computing,Volume 65April 2018,Pages 360-373.

[14] H. T. Jadhav, P. D. Bamane,"Temperature dependent optimal power flow using g-best guided artificial bee colony algorithm", International Journal of Electrical Power \& Energy Systems,Volume 77,May 2016,Pages 7790.

[15] Abdel-Fattah Attia, Ragab A. El Sehiemy, Hany M. Hasanien,"Optimal power flow solution in power systems using a novel Sine-Cosine algorithm",International Journal of Electrical Power \& Energy Systems, Volume 99,July 2018,Pages 331-343.

[16] Ambarish Panda, M. Tripathy, A. K. Barisal, T. Prakash,"A modified bacteria foraging based optimal power flow framework for Hydro-Thermal-Wind generation system in the presence of STATCOM", EnergyVolume 1241 April 2017Pages 720-740

[17] Wenlei Bai, Ibrahim Eke, Kwang Y. Lee," An improved artificial bee colony optimization algorithm based on orthogonal learning for optimal power flow problem", Control Engineering PracticeVolume 61April 2017,Pages 163-172

[18] Belkacem Mahdad, K. Srairi,"Security constrained optimal power flow solution using new adaptive partitioning flower pollination algorithm",Applied Soft Computing,Volume 46,September 2016,Pages 501-522.

[19] P. K. Adhvaryyu, P. K. Chattopadhyay, A. Bhattacharya,"Dynamic optimal power flow of combined heat and power system with Valve-point effect using Krill Herd algorithm", Energy,Volume 12715, May 2017,Pages 756767.

[20] Al-Attar Ali Mohamed, Yahia S. Mohamed, Ahmed A. M. El-Gaafary, Ashraf M. Hemeida,"Optimal power flow using moth swarm algorithm", Electric Power Systems, Volume 142,January 2017,Pages 190-206.

[21] Prerna Singal, A Charan Kumari, Prabha Sharma,"Estimation of Software Development Effort: A Differential Evolution Approach" Procedia Computer Science,Volume 167,2020,Pages 2643-2652.

[22] Veenus Kansal, J. S. Dhillon,"Emended salp swarm algorithm for multiobjective electric power dispatch problem", Applied Soft Computing,Volume 90,May 2020.

[23] Sarath K S, Dr S Sekar,"An Optimal Design of LLC Resonant Converter using FireFly Algorithm based Parameter Optimization Approach",Journal of Computational Mechanics, Power System and Control (JCMPS), Volume 2, Issue 1, January 2019

[24] Subramanyam T C,Tulasi Ram S S,Subrahmanyam J B V,"An Adaptive Chicken Swarm Algorithm to Solve Optimal Power Flow Problem Considering FACTS Device",Journal of Computational Mechanics, Power System and Control (JCMPS), Volume 2, Issue 1, January 2019. 
[25] Harish Kumar V C,Amala Shanthi S,"Hybrid OBL-GO Algorithm to Support Damping Oscillations in Coordinated Control of UPFC and TCSC"Journal of Computational Mechanics, Power System and Control (JCMPS), Volume 3, Issue 1, January 2020.

[26] Sarath K S, Sekar S,"Black Widow Optimization Algorithm: Optimal Designing and modelling and of LLC Resonant Converter",Journal of Computational Mechanics, Power System and Control (JCMPS), Volume 3, Issue 1, January 2020.

[27] Mahendran M V,Vijayan V,"Optimal Sizing and Siting of Distributed Generators by Hybrid Particle Swarm Optimization-Grey Wolf Optimization Algorithm",,Journal of Computational Mechanics, Power System and Control (JCMPS), Volume 3, Issue 1, January 2020 